\title{
Particle-vortex dynamics in noncommutative space
}

\author{
D.H. Correa ${ }^{a *}$, G.S. Lozano ${ }^{b \dagger}$, \\ E.F. Moreno ${ }^{a} \dagger$ and F.A.Schaposnik ${ }^{a \ddagger}$ \\ ${ }^{a}$ Departamento de Física, Universidad Nacional de La Plata \\ C.C. 67, 1900 La Plata, Argentina \\ ${ }^{b}$ Departamento de Física, FCEyN, Universidad de Buenos Aires \\ Pab.1, Ciudad Universitaria, CP 1428, Buenos Aires,Argentina
}

November 5, 2018

\begin{abstract}
We study the problem of a charged particle in the presence of a uniform magnetic field plus a vortex in noncommutative planar space considering the two possible non-commutative extensions of the corresponding Hamiltonian, namely the "fundamental" and the "antifundamental" representations. Using a Fock space formalism we construct eigenfunctions and eigenvalues finding in each case half of the states existing in the ordinary space case. In the limit of $\theta \rightarrow 0$ we recover the two classes of states found in ordinary space, relevant for the study of anyon physics.
\end{abstract}

The Landau and the Aharonov-Bohm problems are the two paradigms of planar quantum mechanics of charged particles in a magnetic field [1]-[国]. Inspired by recent observations concerning the relevance of noncommutative geometry for describing the Quantum Hall effect [3]-[12], both problems have been recently considered in noncommutative space.

Concerning the Landau problem -charged particles in a constant magnetic fieldit was recently analyzed in noncommutative space in [13]-16]. Related discussions

${ }^{*}$ CONICET

$\dagger$ Associated with CONICET

${ }^{\ddagger}$ Associated with CICPBA 
within the framework of the extended Galilean group were presented in [17]- [19]. Regarding the Aharonov-Bohm problem -charged particles in the background of a vortex- some results in noncommutative space were presented in [20]-22].

Adding both magnetic fields, one uniform, the other vortex-like, turns the problem even more interesting. To our knowledge this system was first analyzed and solved in ordinary space in [23], in the context of anyon physics, where particles with exotic statistic are represented by charges pierced by a magnetic flux. In this regard, the problem of two anyons in an external magnetic field is equivalent to that of a particle in the presence of an external magnetic field plus a vortex. From the approach of [23] one concludes that in ordinary space, the eigenstates and spectrum can be found analytically by modifying in a simple but subtle way, the usual Landau problem ladder operator formalism. The presence of the vortex manifests itself in a way simpler than that of the usual Aharonov-Bohm problem.

We shall consider here the generalization of this problem to noncommutative planar space. Namely, we shall solve the Schrödinger equation for a charged particle in the presence of a uniform magnetic field plus a vortex, when space coordinates satisfy

$$
\left[x^{1}, x^{2}\right]=i \theta
$$

It is convenient to introduce complex variables $z$ and $\bar{z}$

$$
z=\frac{1}{\sqrt{2}}\left(x^{1}+i x^{2}\right), \quad \bar{z}=\frac{1}{\sqrt{2}}\left(x^{1}-i x^{2}\right)
$$

and, associated to them, annihilation and creation operators $a$ and $a^{\dagger}$ in the form

$$
a=\frac{1}{\sqrt{2 \theta}}\left(x^{1}+i x^{2}\right), \quad a^{\dagger}=\frac{1}{\sqrt{2 \theta}}\left(x^{1}-i x^{2}\right),
$$

so that (1) becomes

$$
\left[a, a^{\dagger}\right]=1 .
$$

In this way, through the action of $a^{\dagger}$ on the vacuum state $|0\rangle$, eigenstates of the number operator

$$
N=a^{\dagger} a
$$

are generated.With this conventions, derivatives in the Fock space are given by

$$
\partial_{z}=-\frac{1}{\sqrt{\theta}}\left[a^{\dagger},\right], \quad \partial_{\bar{z}}=\frac{1}{\sqrt{\theta}}[a,] .
$$


Some useful formulæ for what follows are

$$
\begin{array}{cl}
a^{q} f(N)=f(q+N) a^{q}, & \left(a^{\dagger}\right)^{q} f(N)=f(N-q)\left(a^{\dagger}\right)^{q} \\
{\left[a^{\dagger}, a^{p}\right]=-p a^{(p-1)}, \quad\left[a,\left(a^{\dagger}\right)^{p}\right]=p\left(a^{\dagger}\right)^{p-1}}
\end{array}
$$

As stated above, we shall discuss the quantum mechanics of a particle in the background of a constant magnetic field plus a magnetic vortex. For the sake of clarity, we first analyze the constant field strength alone and then discuss the effect of adding a vortex field. We define the vector potential components

$$
A_{z}=\frac{1}{\sqrt{2}}\left(A_{1}-i A_{2}\right), \quad A_{\bar{z}}=\frac{1}{\sqrt{2}}\left(A_{1}+i A_{2}\right)
$$

so that the field strength can be written as

$$
F_{z \bar{z}}=\partial_{z} A_{\bar{z}}-\partial_{\bar{z}} A_{z}+i e\left[A_{z}, A_{\bar{z}}\right]
$$

A constant field strength background is given by

$$
F_{z \bar{z}}=i B^{0}
$$

Let us now introduce covariant derivatives. In noncommutative space, even for an Abelian gauge theory one has the possibility of defining them in "fundamental" and "anti-fundamental" representations,

$$
\begin{aligned}
& D_{z} \Psi=\partial_{z} \Psi+i e A_{z} \Psi \quad D_{\bar{z}} \Psi=\partial_{\bar{z}} \Psi+i e A_{\bar{z}} \Psi \quad \text { fundamental } \\
& D_{z} \Psi=\partial_{z} \Psi-i e \Psi A_{z} \quad D_{\bar{z}} \Psi=\partial_{\bar{z}} \Psi-i e \Psi A_{\bar{z}} \quad \text { anti-fundamental } .
\end{aligned}
$$

There is also the possibility of defining the covariant derivative in the adjoint representation, which will not be considered here. We shall first discuss the case of the fundamental representation and then extend the results to the anti-fundamental.

The Schrödinger equation governing the time evolution of the wave function $\Psi$ is given by

$$
-\frac{1}{2 m}\left[D_{z}, D_{\bar{z}}\right]_{+} \Psi=i \frac{\partial \Psi}{\partial t}
$$

where we have defined $[f, g]_{+}=f g+g f$. Then writing

$$
\Psi=\Psi(\bar{z}, z) \exp (-i E t)
$$


one has, for the time-independent Schrödinger equation,

$$
H \Psi(\bar{z}, z)=-\frac{1}{2 m}\left[D_{z}, D_{\bar{z}}\right]_{+} \Psi=E \Psi(\bar{z}, z) .
$$

Let us now construct the angular momentum operator $L$. To this end, it is useful to define the "Eulerian" position operators $w$ and $\bar{w}$, related to the "Lagrangian" ones $(z$ and $\bar{z})$ through [3]

$$
w=z+i e \theta A_{\bar{z}} \quad \bar{w}=\bar{z}-i e \theta A_{z} .
$$

Given the angular momentum operator, defined in ordinary space as

$$
L=z \partial_{z}-\bar{z} \partial_{\bar{z}}
$$

one can see that in noncommutative space the appropriate $L$-operator in the presence of a constant magnetic field $B^{0}$ can be written as [13], 24]

$$
L=w D_{z}-\bar{w} D_{\bar{z}}-\frac{e B^{0}}{2\left(1-e B^{0} \theta\right)}[\bar{w}, w]_{+}-\frac{\theta}{2}\left[D_{\bar{z}}, D_{z}\right]_{+} .
$$

It is important to note that $L$, as defined in (17) is the generalization to noncommutative space of the so called "mechanical angular momentum" 25.

It is convenient at this point to introduce two pairs of creation and annihilation operators, the noncommutative analogue of those developed for the usual Landau problem [26], 23] (for the constant magnetic field case, a related discussion in noncommutative space is presented in [6], [13]-[16]). The actual form of these operators depend on the sign of $e B^{0}$ and, in the present noncommutative case, also on the magnitude of $e B_{0} \theta$. They are defined as

$$
\begin{array}{cc}
c_{1}=\frac{1}{\sqrt{\left|e B^{0}\right|}} D_{z}, & c_{2}=-\frac{1}{\sqrt{\left|e B^{0}\right|}} D_{\bar{z}} \\
d_{1}=\frac{1}{\sqrt{|\kappa|}} w+\operatorname{sgn} \kappa \sqrt{|\kappa|} D_{\bar{z}}, & d_{2}=\frac{1}{\sqrt{|\kappa|}} \bar{w}-\operatorname{sgn} \kappa \sqrt{|\kappa|} D_{z},
\end{array}
$$

where

$$
\kappa=\frac{1-e B^{0} \theta}{e B^{0}}
$$

One can verify that with this definition

$$
\left[c_{1}, c_{2}\right]=\operatorname{sgn}\left(e B^{0}\right), \quad\left[d_{1}, d_{2}\right]=\operatorname{sgn} \kappa, \quad\left[c_{i}, d_{j}\right]=0
$$


Since

$$
c_{2}=c_{1}^{\dagger}, \quad d_{2}=d_{1}^{\dagger},
$$

operators $c_{1}$ and $c_{2}$ will play, depending on the sign of $e B^{0}$ and $\kappa$, the role of creation $\left(c^{\dagger}\right)$ or annihilation $(c)$ operators and the same occurs for the $d_{i}$ 's.

In terms of these operators, the Hamiltonian (14) takes the form

$$
H=\frac{\omega}{2}\left[c, c^{\dagger}\right]_{+}
$$

with

$$
\omega=\frac{\left|e B^{0}\right|}{m}
$$

Concerning the angular momentum, one has to distinguish two regimes,

$$
\begin{array}{lll}
L=\operatorname{sgn} \kappa\left(c^{\dagger} c-d^{\dagger} d\right) & \text { for } & 1-e B^{0} \theta>0, \\
L=-\left(c^{\dagger} c+d^{\dagger} d\right) & \text { for } & 1-e B^{0} \theta<0 .
\end{array}
$$

In both cases, one can easily see that

$$
[H, L]=0
$$

In order to determine the spectrum one has then to find the common eigenfunctions of $H$ and $L$, that is, one has to construct the Fock space of $c$ 's and $d$ 's. We shall now proceed to this construction but for the more general case in which a magnetic vortex is added to the constant magnetic field background. That is, we shall consider a field strength of the form

$$
F_{z \bar{z}}=i B^{0}-i \frac{\alpha}{e \theta}|0\rangle\langle 0|
$$

Here $|0\rangle\langle 0|$ is the projector onto the state $|0\rangle$ annihilated by operator $a$ defined in eq.(3). One can easily see that in the $\theta \rightarrow 0$ limit, the second term in (27) goes, in configuration space, to a delta function corresponding to a singular vortex at the origin, with flux related to the real parameter $\alpha$ according to [27]-30]

$$
\Phi^{v o r}=2 \pi i \theta \operatorname{Tr} F_{z \bar{z}}^{v o r}=\frac{2 \pi}{e} \alpha
$$

As in the constant field case, we can introduce operators $c_{i}$ and $d_{i}$ now taking the form 


$$
\begin{array}{cc}
c_{1}=\frac{1}{\sqrt{\left|e B^{0}\right|}} D_{z}[A], & c_{2}=-\frac{1}{\sqrt{\left|e B^{0}\right|}} D_{\bar{z}}[A] \\
d_{1}=\frac{1}{\sqrt{|\kappa|}} w[A]+\operatorname{sgn} \kappa \sqrt{|\kappa|} D_{\bar{z}}[A], & d_{2}=\frac{1}{\sqrt{|\kappa|}} \bar{w}[A]-\operatorname{sgn} \kappa \sqrt{|\kappa|} D_{z}[A],
\end{array}
$$

where $\kappa$ is still given by (20) (i.e., depending only on the constant part of the magnetic field). Concerning the operator algebra, one has, instead of (21),

$$
\begin{aligned}
{\left[c_{1}, c_{2}\right] } & =\operatorname{sgn}\left(e B^{0}\right)-\frac{\alpha}{\theta\left|e B^{0}\right|}|0\rangle\langle 0|, \\
{\left[d_{1}, d_{2}\right] } & =\operatorname{sgn} \kappa+\frac{\alpha}{\theta\left|e B^{0}\left(1-e B^{0} \theta\right)\right|}|0\rangle\langle 0|, \\
{\left[c_{1}, d_{1}\right] } & =\frac{\alpha}{e B^{0} \theta \sqrt{\left|1-e B^{0} \theta\right|}|0\rangle\langle 0|,} \\
{\left[c_{2}, d_{2}\right] } & =-\frac{\alpha}{e B^{0} \theta \sqrt{\left|1-e B^{0} \theta\right|}}|0\rangle\langle 0|, \\
{\left[c_{1}, d_{2}\right] } & =\left[c_{2}, d_{1}\right]=0 .
\end{aligned}
$$

Note that for those states $\chi$ such that

$$
|0\rangle\langle 0| \chi=0
$$

this algebra coincides with that defined in (21). We shall call $\mathcal{P}_{0}$ the subspace of states satisfying (33). This condition, in the commutative space limit becomes the "hard-core condition" since it corresponds to the vanishing of wave functions at the origin. Moreover, in the $\theta \rightarrow 0$ limit, the algebra (32) coincides with that obtained in [23] (except that in this last reference it is presented in the singular gauge).

Again, depending on the signs and magnitudes of $e B^{0}$ and $\theta$ the $c_{i}$ 's and $d_{i}$ 's will act as creation or annihilation operators. As in the constant magnetic field case, the Hamiltonian $H$ and angular momentum $L$ can be written in the form (23) and (17), provided states are restricted to $\mathcal{P}_{0}$.

At this point we have to write an explicit expression for the vector potential leading to a field strength $F_{z \bar{z}}$ as given by (27). One can see that a possible choice is

$$
A_{z}=\frac{i}{\sqrt{\theta}} g(N) a^{\dagger}, \quad A_{\bar{z}}=-\frac{i}{\sqrt{\theta}} a g(N)
$$


with

$$
g(N)=-\frac{1}{e}\left(1-\sqrt{1-e B^{0} \theta+\frac{\alpha}{N}}\right) .
$$

Here, we have proposed a form which is valid for $1-e B^{0} \theta>0$ and positive $\alpha$. Other possibilities can be handled similarly.

Given the Hamiltonian (23) and angular momentum (25), we shall now construct the Fock space associated to the operators $N_{c}=c^{\dagger} c$ and $N_{d}=d^{\dagger} d$. Let us start by considering a state $\chi$ such that

$$
c \chi=0
$$

Then $\chi$ is an eigenstate of the Hamiltonian

$$
H \chi=\frac{\omega}{2} \chi
$$

In order to make $\chi$ also an eigenstate of $L$, we propose the following ansatz

$$
\chi\left(a, a^{\dagger}\right)=a^{\dagger n} h(N)
$$

Note that in the commutative limit $a^{\dagger n}$ can be connected to $\exp (-i n \varphi)$, an eigenfunction of the canonical angular momentum with eigenvalue $-n$.

One can see that $\chi \in \mathcal{P}_{0}$ provided $n>0$. Applying $L$ in the form (25), one sees that the ansatz is consistent only for $0<e B^{0} \theta<1$; we shall study other regimes later on. One has,

$$
L \chi=-(n+\bar{\alpha}) \chi, \quad 0<e B^{0} \theta<1
$$

where

$$
\bar{\alpha}=\frac{\alpha}{1-e B^{0} \theta} .
$$

One can determine $h(N)$ starting from $n=1$. The corresponding $\chi$ eigenstate will be denoted as $\chi_{01}$,

$$
\chi_{01}=a^{\dagger} h(N) .
$$

Calling

$$
h_{m}=\langle m|h(N)| m\rangle \text {, }
$$

one finds

$$
h_{m}=\left(\frac{1}{2 \pi \theta} \frac{\Gamma(m+2+\bar{\alpha})}{(m+1) ! \Gamma(2+\bar{\alpha})}\left(e B^{0} \theta\right)^{2+\bar{\alpha}}\left(1-e B^{0} \theta\right)^{m}\right)^{1 / 2}
$$


We can express eigenfunction (41) in terms of ordinary functions using the connection $|m\rangle\langle m| \rightarrow 2(-1)^{m} L_{m}\left(2 r^{2} / \theta\right) \exp \left(-r^{2} / \theta\right)$. We can then explicitly write

$$
\chi_{01}(z, \bar{z})=\bar{z} * \frac{1}{\sqrt{\theta}} \sum_{m} 2(-1)^{m} h_{m} L_{m}\left(2 r^{2} / \theta\right) \exp \left(-r^{2} / \theta\right)
$$

A tower of states with increasing energy can be constructed from $\chi_{01}$ by acting with $c^{\dagger}$ and $d^{\dagger}$,

$$
\chi_{k l}=\left(c^{\dagger}\right)^{k}\left(d^{\dagger}\right)^{l-1} \chi_{01}, \quad l>k .
$$

Being $l>k$, one can see that $\chi_{k l} \in \mathcal{P}_{0}$. The corresponding energy and angular momentum eigenvalues are

$$
\begin{array}{rlr}
E_{k} & =\omega\left(k+\frac{1}{2}\right) & \\
& 0<e B^{0} \theta<1 \\
L_{k l} & =(k-l-\bar{\alpha}) .
\end{array}
$$

It is not difficult to see that in the commutative $(\theta \rightarrow 0)$ limit, eigenstate (44), and consequently all the tower (45), coincide with the corresponding "class II" states found in [23].

Inspired by the ordinary space results, one could try to construct a second tower of ("class I") states using, instead of (36), the condition

$$
d \eta=0
$$

In contrast with what happens in ordinary space [23], in noncommutative space one finds that there is no solution belonging to $\mathcal{P}_{0}$ in the region $0<e B^{0} \theta<1$.

Let us now study the region $e B^{0} \theta<0$. In this case $\kappa<0$ and then $c_{1}, c_{2}$ (and also $\left.d_{1}, d_{2}\right)$ interchange their roles of creation and annihilation operators. Now, non-trivial solutions $\eta$ can be found in this region if one starts from the condition

$$
d \eta=0
$$

Eigenstates and eigenvalues can be obtained from a state $\eta_{10}$ of the form

$$
\eta_{10}=a^{\dagger} f(N)
$$


with $f(N)$ given by

$$
f_{m}=\left(\frac{1}{2 \pi \theta} \frac{\Gamma(m+2+\bar{\alpha})}{(m+1) ! \Gamma(2+\bar{\alpha})} \frac{\left(-e B^{0} \theta\right)^{2+\alpha}}{\left(1-e B^{0} \theta\right)^{m-2-\alpha}}\right)^{1 / 2} .
$$

The tower of solutions with increasing energy is now given by

$$
\eta_{k l}=\left(c^{\dagger}\right)^{k-1}\left(d^{\dagger}\right)^{l} \eta_{10}, \quad k>l
$$

and eigenvalues take the form

$$
\begin{array}{rlrl}
E_{k} & =\omega\left(k+\frac{1}{2}-\bar{\alpha}\right) & \\
e B^{0} \theta<0, & \\
L_{k l} & =(l-k-\bar{\alpha}) .
\end{array}
$$

As in the previous case, eigenstates and eigenvalues coincide, in the $\theta \rightarrow 0$ limit with those called "class I" in ordinary space 23.

We have not found an acceptable ansatz for the $e B^{0} \theta>1$ region. The problem is the following: in order to treat this region, one has to modify the ansatz for the vector potential, adding to $A_{z}$ as given by (8) a term proportional to $z$ (and a term proportional to $\bar{z}$ in $A_{\bar{z}}$ ). Now, with this form for the vector potential, we were not able to construct the tower of eigenstates belonging to $\mathcal{P}_{0}$. It is interesting to note that this region does not exist in the commutative $\theta \rightarrow 0$ limit.

The discussion above corresponds to the fundamental representation as defined by (11). The analysis for the anti-fundamental representation follows the same steps. One defines operators $c_{i}$ and $d_{i}$ as in (29)-(30) but with the covariant position and derivative operators in the anti-fundamental, according to (11). Again, we have to restrict states to a subspace $\tilde{\mathcal{P}}_{0}$ such that, on it, the operator algebra reduces to the canonical one, eq.(21). The condition on states reads now

$$
\tilde{\chi} \in \tilde{\mathcal{P}}_{0} \rightarrow \tilde{\chi}|0\rangle\langle 0|=0, \quad
$$

One finds for the lowest state

$$
\tilde{\chi}_{01}=s(N) a
$$


with $s(N)$ adjusted so that $c \tilde{\chi}_{01}=0$. From this state, one construct a tower of states through

$$
\begin{array}{rlrl}
\tilde{\chi}_{k l} & =\left(c^{\dagger}\right)^{k}\left(d^{\dagger}\right)^{l-1} \tilde{\chi}_{01}, \quad l>k, \\
E_{k} & =\omega\left(k+\frac{1}{2}\right) & & 0<e B^{0} \theta<1 \\
L_{k l} & =-(k-l-\bar{\alpha}) . &
\end{array}
$$

Analogously, from

$$
\tilde{\eta}_{10}=t(N) a
$$

one has

$$
\begin{array}{rlrl}
\tilde{\eta}_{k l} & =\left(c^{\dagger}\right)^{k-1}\left(d^{\dagger}\right)^{l} \tilde{\eta}_{10}, \quad k>l, & \\
E_{k} & =\omega\left(k+\frac{1}{2}-\bar{\alpha}\right) & & e B^{0} \theta<0 \\
L_{k l} & =-(l-k-\bar{\alpha}) . &
\end{array}
$$

Note that these results coincide with those for the fundamental representation except for the sign in the angular momentum eigenvalues.

It is interesting at this point to connect our results with those obtained in ordinary (commutative) space by Johnson and Canright [23] in their discussion of the physics of two anyons in a uniform magnetic field. Using the analogue of operators $c_{i}$ and $d_{i}$, these authors construct two classes of eigenstates, one for which the energy does depend on the vortex flux $\alpha$ (class I) and the other which exhibits an $\alpha$-independent energy (class II). Concerning the angular momentum, the two classes have opposite sign eigenvalues. While in the ordinary Landau problem all states have energy depending only on the integer Landau index, when particles have attached a flux tube of strength $\alpha$, class I states (which, according to their angular momentum eigenvalue circulate in a classically "incorrect" direction) have an energy which is shifted by $\alpha$.

How this scenario is modified in noncommutative space? Only one class of states can be constructed both for the fundamental and the antifundamental representations. Indeed, in the fundamental, for uniform magnetic field in the range $0<e B^{0}<1 / \theta$, only class II $(\alpha$-independent energy) states exist while for a range 
$e B^{0}<0$ the only possible states are class I type with $\alpha$-dependent energy; concerning the antifundamental representation, the same phenomenon happens. Now, in the commutative limit $(\theta \rightarrow 0)$ the fundamental representation (with charge $e$ ) and the anti-fundamental representation (with charge $-e$ ) merge, covering the whole range of values of $e B^{0}$ so that we have both classes of solutions in both regions, thus recovering Johnson and Canright result.

We have then solved explicitly the spectrum of a Hamiltonian describing a charged particle in the presence of a background magnetic field and a vortex in both, the fundamental and the anti-fundamental representations, for the case $1-e B^{0} \theta>0$. We have shown that the "hard-core" condition plays an important role in the selection of the allowed states, giving only negative (positive) angular momentum eigenstates for the fundamental (anti-fundamental) representation. It is well known that the issue of hard-core condition is related to the problem of self-adjoint extensions of the Hamiltonian in commutative space. It would be interesting to explore these ideas in the noncommutative setting and to study the connection between the parameter $\theta$ and the self-adjoint parameter, both dimensionful. Like in the commutative space, one would probably need to solve in this case the complete second-order Schrödinger differential equation.

As we mentioned in the introduction, in commutative space the problem of a particle on the background of a vortex is equivalent to the two-anyon problem. In turn, the problem of ayons can be analyzed as the quantum mechanics of ordinary particles interacting with gauge fields whose dynamics is governed by a ChernSimons term [31]-32]. As far as we know, this line of research has not been yet implemented in noncommutative space and it would be worthwhile to advance along this line. Also, it would be interesting to analyze this problem within the pathintegral approach developed in [33].

It is not clear if our approach can be extended to the region $1-e B^{0} \theta<0$, which does not have a commutative limit. We hope to report on these issues elsewhere.

Acknowledgements: This work partially supported by UNLP, CICBA, CONICET (PIP 4330/96), ANPCYT (PICT 03-05179), Argentina. G.S.L. and E.F.M. are partially supported by Fundación Antorchas, Argentina. D.H.C. was partially sup- 
ported through an ANPCYT fellowship.

\section{References}

[1] R. Jackiw, Topics in Planar Physics, Banff NATO ASI 0191 (1989) 240.

[2] R. Jackiw, Annals Phys. (N.Y.) 201 (1990) 83.

[3] L. Susskind, hep-th/0101029.

[4] A. P. Polychronakos, JHEP 0104, 011 (2001) hep-th/0103013].

[5] S. S. Gubser and M. Rangamani, JHEP 0105, 041 (2001) hep-th/0012155].

[6] S. Hellerman and M. van Raamsdonk, hep-th/0103179.

[7] B. Lee, K. Moon and C. Rim, hep-th/0105127.

[8] B. Morariu and A. P. Polychronakos, JHEP 0107, 006 (2001) [hep-th/0106072].

[9] C. Duval and P. A. Horvathy, hep-th/0106089.

[10] S. Hellerman and L. Susskind, hep-th/0107200.

[11] B. Freivogel, L. Susskind and N. Toumbas, hep-th/0108076.

[12] A. El Rhalami, E. M. Sahraoui and E. H. Saidi, hep-th/0108096.

[13] V.P. Nair and A. Polychronakos, Phys. Lett. B505 (2001) 267.

[14] A. Jellal, hep-th/0105303.

[15] J. Gamboa, M. Loewe, F. Méndez and J.C. Rojas, hep-th/0106125.

[16] S. Bellucci, A. Nersessian and C. Sochichiu, hep-th/0106138.

[17] J. Lukierski, P. Stichel, W. Zakrzewski, Ann. of Phys. (N.Y.) 260 (1997) 294.

[18] C. Duval and P. A. Horvathy, Phys. Lett. B479 (2000) 284

[19] C. Duval and P. A. Horvathy, cond-mat/0101449. 
[20] M. Chaichian, A. Demichev, P. Presnajder, M. M. Sheikh-Jabbari and A. Tureanu, hep-th/0012175.

[21] M. Chaichian, A. Demichev, P. Presnajder, M. M. Sheikh-Jabbari and A. Tureanu, hep-th/0101209.

[22] J. Gamboa, M. Loewe and J. C. Rojas, hep-th/0101081.

[23] M.D. Johnson and G.S. Canright, Phys. Rev. B 41 (1990) 6870.

[24] D. Bak, S.K. Kim, K.-S. Soh and J.H. Yee, Phys. Rev. D64 (2001) 025018.

[25] R. Jackiw and A.N. Redlich, Phys. Rev. Lett. 50 (1983) 555.

[26] A.H. MacDonald, Phys. Rev. B 30 (1984) 3550.

[27] A. Polychronakos, Phys. Lett. B495 (2000) 407.

[28] D.J. Gross and N. Nekrasov, JHEP 0007 (2000) 034; JHEP 0010 (2000) 021.

[29] D.P. Jaktar, G. Mandal and S.R. Wadia, JHEP 0009 (2000) 018.

[30] J.A. Harvey, P. Kraus and F. Larsen, JHEP 0012 (2000) 024.

[31] E. Fradkin, Field Theories of Condensed Matter systems, Frontiers in Physics, Addison-Wesley, New York, 1991.

[32] See R.Jackiw in Diverse Topics in Theoretical and Mathematical Physics World Sci. Singapore, 1995, pp 465-514 and references therein.

[33] H. Christiansen and F.A. Schaposnik, hep-th/0106181 